\title{
SOME REMARKS ON A PAPER BY R. H. BRUCK
}

\author{
TREVOR EVANS
}

Introduction. In a recent paper [2] R. H. Bruck has introduced the concept of right neoring and discussed some properties of these systems. In particular, he has considered analogues of certain properties of the ring of integers. This paper is essentially a commentary on Bruck's paper and we generalize some of his results as follows. The construction of the universal right neoring in [2] is applied to the free monogenic $\mathfrak{B}$-loop in any subvariety $\mathfrak{B}$ of the variety of loops and a complete analogue of Theorem 4.1 of [2] is obtained for any one of these subvarieties. Then, using a result similar to those obtained in [5], it is shown that this construction yields uncountably many right neorings with an identity which generates the additive loop of the right neoring. Conversely, every right neoring with an identity which generates its additive loop can be obtained from a free monogenic $\mathfrak{B}$-loop by the above construction. Each of these right neorings has some properties resembling those of the ring of integers. One possible answer is given to the question raised by Bruck concerning the existence of universal right neorings with free additive loop of arbitrary rank. A brief proof is given, using the results of $[4 ; 5]$, of the cancellation properties of the monogenic universal right neoring. Finally, we discuss briefly the relationship between right neorings and the logarithmetics of Etherington.

Preliminaries. By a variety of algebras we mean an equationally defined class of abstract algebras in which the operations are finite in number and finitary in scope. However, the number of identical relations defining the variety may be infinite. A subvariety $\subseteq$ of a variety $\mathfrak{B}$ is a variety with the same set of operations as $\mathfrak{B}$ and such that the identities defining $\mathfrak{B}$ are a subset of those defining $\mathfrak{S}$. The class of loops is a variety if we use the definition of a loop in terms of three operations given in [4].

In any variety of algebras $\mathfrak{B}$, we have the free algebra $F_{n}(\mathfrak{B})$ on $n$ generators. This can be defined as follows. An algebra $V \in \mathfrak{B}$ is called the free algebra $F_{n}(\mathfrak{B})$ if any mapping of the $n$ generators of $V$ into any algebra $W \in \mathfrak{B}$ can be extended to a homomorphism of $V$ into $W$. Such an algebra, if it exists, is unique to within isomorphism. An explicit construction of $F_{n}(\mathfrak{B})$ can be obtained in the usual way in

Presented to the Society, October 22, 1955; received by the editors April 25, 1955. 
terms of words in the generators. An important property of $F_{n}(\mathfrak{B})$ is that any mapping of its generators into itself can be extended to an endomorphism. This property does not completely characterize $F_{n}(\mathfrak{B})$ in the variety $\mathfrak{B}$ since an algebra in $\mathfrak{B}$ with this property could be the free algebra on $n$ generators in some subvariety of $\mathfrak{B}$. We shall use later the following theorem.

THEOREM 1. Let $V$ be a $\mathfrak{B}$-algebra with the property that any mapping of the generators of $V$ into $V$ can be extended to an endomorphism. Then $V$ is isomorphic to $F_{n}(\mathfrak{S})$ for some subvariety $\mathfrak{S}$ of $\mathfrak{B}$.

Proof. Let $\mathfrak{S}$ be the subvariety of $\mathfrak{B}$ consisting of all $\mathfrak{B}$-algebras satisfying all the identical relations which $V$ satisfies. Then $V$ is the free algebra on $n$ generators (for some $n$ ) of the variety $\mathfrak{S}$ since any relations which the generators of $V$ satisfy are satisfied identically in $V$ and hence in every algebra in $\mathfrak{S}$. Thus any mapping of the generators of $V$ into an algebra in $\mathfrak{S}$ can be extended to a homomorphism of $V$ into this algebra.

In any $\mathfrak{B}$-algebra, an endomorphism is completely determined by the images of the generators and, as we have seen, in $F_{n}(\mathfrak{B})$ any set of $n$ elements can be the images of the generators under an endomorphism. This gives the following complete generalization of Lemma 3.1 of [2].

TheOREM 2. Let $V$ be a $\mathfrak{B}$-algebra generated by $g_{1}, g_{2}, \cdots, g_{n}$ and let $\theta$ be the homomorphism $a_{i} \rightarrow g_{i}$ of the free $\mathfrak{B}$-algebra $F_{n}(\mathfrak{B})$ generated by $a_{1}, a_{2}, \cdots, a_{n}$ onto $V$. Then, for every endomorphism $\alpha$ of $V$, $\beta \theta=\theta \alpha$ where $\beta$ is any endomorphism of $F_{n}(\mathfrak{B})$ such that $a_{i} \beta \theta=g_{i} \alpha$.

In a variety $\mathfrak{B}$ it may happen that restrictions on $n$ imply that $F_{n}(\mathfrak{B})$ is the free algebra of some subvariety of $\mathfrak{B}$. For example, if $\mathbb{B}$ is the variety of groups and $\mathscr{A}$ the subvariety of abelian groups, then $F_{1}(\mathfrak{S}) \cong F_{1}(\mathfrak{A})$. Or, if $\mathfrak{M}$ is the variety of Moufang loops and $\mathbb{S}$ the subvariety of groups, then $F_{2}(\mathfrak{M}) \cong F_{2}(\mathfrak{S})$. However, although some identifications of this type may occur in the set of all $F_{1}(\mathfrak{B})$ where $\mathfrak{B}$ ranges over all subvarieties of the variety $\mathbb{B}$ of groupoids or the variety $\mathbb{R}$ of loops, we still have the following theorem.

THEOREM 3.

(a) There are uncountably many nonisomorphic groupoids of the form $F_{1}(\mathfrak{B})$ where $\mathfrak{B}$ is a subvariety of $\mathfrak{B}$.

(b) There are uncountably many nonisomorphic loops of the form $F_{1}(\mathfrak{B})$ where $\mathfrak{B}$ is a subvariety of $\mathfrak{R}$.

Proof. The existence of monogenic groupoids satisfying all but one 
of an infinite set of identical relations as shown in $[6, p .345]$ is sufficient to prove part (a). However, part (b) cannot be proved in this manner by reference to Theorem 2.3 of [6] since the loops constructed in the proof of that theorem are not monogenic. It is comparatively easy to remedy this by exhibiting an infinite set of identical relations such that monogenic loops exist satisfying all but one of the relations. The following proof (due to the referee) is briefer.

In $[1$, p. 450$]$, Artzy has constructed a class of loops such that each loop is uniquely determined to within isomorphism by a singlevalued function on the integers, this function satisfying a certain condition. From the construction given by Artzy it can be seen that there are uncountably many different functions of this type and hence uncountably many nonisomorphic loops in the class. Bruck has shown $[2$, p. 57] that each of these loops is monogenic and is the additive loop of a neofield. By Theorem 6 in the next section such a loop is the free loop in some variety of loops. This proves part (b) of the theorem.

Right neorings. As defined in [2], a right neoring $R$ is a system with two binary operations $(+),(\cdot)$ such that $R$ is a loop with respect to $(+)$ with zero $0, R$ is closed with respect to $(\cdot)$ and $x 0=0$, and $(x+y) z=x z+y z$ for all $x, y, z$ in $R$. A simple consequence of these axioms is that $0 x=0$ for all $x$ in $R$. If $R$ has a left identity 1 which generates the additive loop of $R$, then 1 is actually a two-sided identity and multiplication in $R$ is associative (Theorem 2.1 of [2]). In this section we shall generalize Bruck's construction of a universal right neoring and show that our construction actually gives all right neorings having a left identity which generates the additive loop of the right neoring.

Let $\mathfrak{B}$ be any subvariety of the variety of loops $\mathfrak{R}$. Consider the loop $F_{1}(\mathfrak{B})$ with the loop operation written as addition and let the generator of $F_{1}(\mathfrak{B})$ be written as 1 . Let $\phi_{x}$ be the endomorphism of $F_{1}(\mathfrak{B})$ which maps 1 in to the element $x$ of $F_{1}(\mathfrak{B})$. Define a multiplication $x y$ in $F_{1}(\mathfrak{B})$ by $x y=x \phi_{y}$. Then, exactly as in [2], we obtain a right neoring with an associative multiplication and having 1 as a two-sided identity. We shall denote this right neoring by $R_{1}(\mathfrak{B})$. The construction given in [2] is the special case $\mathfrak{B}=\mathfrak{R}$ of our construction. Theorem 4.1 of [2] becomes, in this general situation, the following.

THEOREM 4. Let $R$ be a right neoring with left identity 1 such that 1 generates the additive loop $R^{+}$of $R$. Then, if $R^{+}$belongs to the subvariety $\mathfrak{B}$ of $\mathfrak{R}$, the homomorphism $\theta$ of $F_{1}(\mathfrak{B})$ onto $R^{+}$given by $1 \theta=1$ induces a homomorphism of the right neoring $R_{1}(\mathfrak{B})$ onto the right neoring $R$. 
Proof. This is the same as the proof of Theorem 4.1 in [2] if Lemma 3.1 of that paper is replaced by our Theorem 2 . We note that this theorem also implies that 1 is a two-sided identity and that multiplication in $R$ is associative.

Combining the preceding construction of right neorings $R_{1}(\mathfrak{B})$ with Theorem 3 we obtain a theorem giving the number of distinct right neorings of the type described in Theorem 4.

THEOREM 5. There are uncountably many nonisomorphic associative right neorings with a two-sided identity which generates the additive loop.

We thus have a means of constructing a large class of right neorings, namely, the $R_{1}(\mathfrak{B})$ for subvarieties $\mathfrak{B}$ of $\mathfrak{R}$. The question arises, what are the other right neorings having a left identity which generates the additive loop? By Theorem 4.1 of [2] (our Theorem 4 with $\mathfrak{B}=\mathfrak{l})$, any such right neoring is a homomorphic image of $R_{1}(\mathfrak{l})$. The next theorem shows that any such homomorphic image is necessarily an $R_{1}(\mathfrak{B})$ for some subvariety $\mathfrak{B}$ of $\mathfrak{R}$. Hence our construction of the $R_{1}(\mathfrak{B})$ includes all right neorings having a left identity such that this left identity generates the additive loop of the right neoring.

THEOREM 6. Let $R$ be a right neoring with a left identity 1 such that 1 generates $R^{+}$, the additive loop of $R$. Then $R$ is isomorphic to $R_{1}(\mathfrak{B})$ for some subvariety $\mathfrak{B}$ of $\mathfrak{R}$.

Proof. The right distributive law in $R$ implies that the mapping $\phi_{y}$ defined by $x \phi_{y}=x y$ is an endomorphism of $R^{+}$into itself for any $y$ in $R$. Since 1 is a left identity in $R, y=1 \cdot y=1 \phi_{y}$. Hence there is an endomorphism of $R^{+}$mapping its generator into any element. In other words, any mapping of the generator 1 of $R^{+}$into $R^{+}$can be extended to an endomorphism of $R^{+}$. By Theorem 1, this implies that $R^{+}$is isomorphic to $F_{1}(\mathfrak{B})$ for some subvariety $\mathfrak{B}$ of $\mathfrak{R}$.

Since multiplication $x y$ in $R$ has the property $x y=x \phi_{y}$ where $\phi_{y}$ is the endomorphism of $F_{1}(\mathfrak{B})\left(=R^{+}\right)$which maps 1 into $y$, the right neoring $R$ is thus $R_{1}(\mathfrak{B})$.

This completes the proof. We mention briefly another approach. The kernel $K$ of the mapping of $R_{1}(\mathfrak{l})$ onto $R$ defined by $1 \rightarrow 1$ is a normal subloop of $F_{1}(\Omega)$. It is actually a fully invariant normal subloop of $F_{1}(\Re)$ since $K \cdot F_{1}(\Omega) \subset K$ (see Lemma 4.3, [2]) so that $K$ is mapped into itself by all endomorphisms of $F_{1}(\Omega)$. Thus $K$ is a word subloop of $F_{1}(\Omega)$ and the quotient loop $F_{1}(L) / K$ is generated by 1 and satisfies the identical relations arising from equating to 0 the words which generate $K$. Hence $R$ is the monogenic free $\mathfrak{B}$-loop for some $\mathfrak{B C}$, where $\mathfrak{B}$ is determined by the words generating $K$. In view of 
the remarks preceding Theorem $4, K$ may be any fully invariant normal subloop of $F_{1}(R)$ showing that condition (b) of Lemma 4.3 of $[2]$ is redundant when the additive loop of the right neoring is $F_{1}(\mathbb{R})$. In fact, the same reasoning shows that condition (b) is redundant whenever the right neoring $(R,+, \cdot)$ (in Bruck's notation) has a left identity which generates the additive loop. A direct proof is possible of a more general result than this, namely, that if $x, y, k$ are three elements of the monogenic loop $F_{1}(\mathfrak{B})$ then $x(y+k)=x y+k^{\prime}$ for some $k^{\prime}$ in the normal subloop of $F_{1}(\mathfrak{B})$ generated by $k$. Here, right multiplication by an element denotes as usual the endomorphism sending 1 into that element.

It is seen then that the study of right neorings with a left identity which generates the additive loop is equivalent to the study of either fully invariant normal subloops of the free monogenic loop or of free monogenic $\mathfrak{B}$-loops for any subvariety $\mathfrak{B}$ of the variety of loops. One property of $R_{1}(\mathfrak{R})$ which is true for any $R_{1}(\mathfrak{B})$ is that given in Lemma 4.2 of [2]. Namely, if $G$ is a subloop of $F_{1}(\mathfrak{B})$, the $G$ is a subneoring of $R_{1}(\mathfrak{B})$. The proof is the same as that for $\mathfrak{B}=\mathfrak{R}$ given in $[2, \mathrm{p} .54]$.

In the proof of the cancellation laws for $R_{1}(\Omega)$ given in [2], the proof that $z x=z y, z \neq 0$, implies $x=y$ is only sketched since a complete proof along the same lines would be very long. However, a brief proof of both cancellation laws is possible using the results of [4] and [5] and we shall give this now. Of course, this proof is only short because the tedious consideration of cases has already been done in these earlier papers.

Let $x, y, z$ be elements in $F_{1}(\mathbb{R})$ and let them be given by the normal forms $u(1), v(1), w(1)$ respectively. Then the cancellation law $x z=y z, z \neq 0, \Rightarrow x=y$ is equivalent to the statement that, if $w(1) \neq 0$, then $u(w(1))=v(w(1)) \Rightarrow u(1)=v(1)$. Similarly, the cancellation law $z x=z y, z \neq 0, \Rightarrow x=y$ is equivalent to the statement that, if $w(1) \neq 0$, then $w(u(1))=w(v(1)) \Rightarrow u(1)=v(1)$. These equivalences follow from the fact that under the endomorphism $1 \rightarrow t$ of $F_{1}(\mathfrak{R})$, the word $p(1)$ is mapped into $p(t)$.

Our proof is actually an extension of that of Lemma 2 in [5]. Let $p(1), q(1)$ be two normal forms in $F_{1}(\mathfrak{R})$, neither of which is the zero element. Now, if $q(1)$ is not a repeated inverse of 1 , the word $p(q(1))$ is necessarily normal, for an elementary reduction of $p(q(1))$ would imply that an elementary reduction is possible in either $q(1)$ or $p(1)$ whereas these words are normal. If $q(1)$ is a repeated left (right)inverse of 1 and $p(1)$ contains no components which are repeated right (left)-inverses of 1 , again $p(q(1))$ is normal, for the same reason.

Hence, in both these cases, if $r(1)$ is another normal word, not 
equal to $q(1)$, then $p(q(1)) \neq p(r(1))$, for two normal forms are equal if and only if they are identical. Also, if $s(1)$ is a normal form not equal to $p(1)$, then $p(q(1)) \neq s(q(1))$.

The only possibility for $p(q(1))$ left to consider is when $q(1)$ is a repeated left (right)-inverse and some of the components of $p(1)$ are repeated right (left)-inverses. This case is treated fully in Lemma 2, [5]. Again, we reach the conclusion that $r(1) \neq q(1)$ implies that $p(q(1)) \neq p(r(1))$ and $s(1) \neq p(1)$ implies $p(q(1)) \neq s(q(1))$.

Universal right neorings. The right neoring $R_{1}(\mathbb{R})$ is universal in the sense that any right neoring with a left identity which generates the additive loop is a homomorphic image of $R_{1}(\mathfrak{R})$. Bruck raises the question of whether there exist universal right neorings with additive loops free of arbitrary rank. Since a right neoring can be constructed, having the free $\mathfrak{B}$-loop on $n$ generators as additive loop for every mapping of the elements of the loop into the set of all endomorphisms (with the restriction that the zero element corresponds to the zero endomorphism) it seems unlikely that a universal right neoring exists for the class of all right neorings having the free $\mathfrak{B}$-loop on $n$ generators as additive loop.

However, we can obtain universal right neorings for some classes of right neorings having $F_{n}(\mathfrak{B})$, the free $\mathfrak{B}$-loop on $n$ generators, as additive loop. One example is a direct generalization of $R_{1}(\mathfrak{B})$. Let $g_{1}, g_{2}, g_{3}, \cdots$ be the generators of $F_{n}(\mathfrak{B})$ ( $n$ may be infinite) and let $\phi_{x}$ be the endomorphism of $F_{n}(\mathfrak{B})$ which maps every $g_{i}$ onto the element $x$ of $F_{n}(\mathfrak{B})$. Define a multiplication $x y$ in $F_{n}(V)$ by $x y=x \phi_{y}$. Then $F_{n}(\mathfrak{B})$ becomes a right neoring which we shall denote by $R_{n}(\mathfrak{B})$. Multiplication in $R_{n}(\mathfrak{B})$ is associative and the elements $g_{i}$ are left identities with respect to multiplication. The right neoring $R_{n}(\mathfrak{B})$ is universal in the sense that any right neoring $R$ with $n$ left identities such that these left identities generate $R^{+}$, the additive loop of $R$, is a homomorphic image of $R_{n}(\mathfrak{B})$ where $\mathfrak{B}$ is a variety of loops containing $R$. The proof of this is the same as the proof of Theorem 4 and Theorem 4.1 of [2].

Logarithmetics. In a series of papers (see [3] and the references given there) Etherington has defined and investigated the basic properties of logarithmetics. Originally defined for a linear algebra, the logarithmetic of this algebra is essentially the algebraic system of all exponents of a general element of the algebra with addition and multiplication defined in the usual way. The resulting system resembles a left neoring with an identity which generates the additive system, the difference being that the additive system is a groupoid 
and not a loop. The logarithmetics of quasigroups and loops have been studied by $H$. Popova (see [7] and the references given there) and in the latter case the resulting algebra is actually a left neoring with an identity which generates the additive loop. In this section we discuss this connection between neorings and logarithmetics.

Let $G$ be a multiplicative groupoid, with or without an identity element, and let $x$ denote an element of $G$. We introduce exponents, which are elements of the free additive groupoid generated by 1 , into $G$ by (i) $x^{1}=x$, (ii) $x^{m+n}=x^{m} \cdot x^{n}$. If $G$ has an identity we introduce 0 into the system of exponents and define $x^{0}=e$, the identity of $G$. Multiplication is introduced into the additive groupoid of exponents by $x^{m \cdot n}=\left(x^{m}\right)^{n}$.

Now let $S$ be a subset of the elements of $G$. Etherington defines the logarithmetic of $S$, which we shall denote by $L(S)$, to be the algebra of all exponents, that is, all words in 1 with respect to $(t)$ and $(\cdot)$, with equality $m=n$ between exponents $m, n$ if and only if $x^{m}=x^{n}$ for every element $x$ in $S$. Important special cases are when $S$ consists of a single element in which case the resulting algebra is called the logarithmetic of the element and when $S$ consists of the whole of $G$ in which case the algebra is called the logarithmetic of the groupoid. It is clear that a logarithmetic $L(S)$ is an algebraic system with two binary operations $(+),(\cdot)$ such that additively the system is generated by one element 1 . For any two additive words $u(1), v(1)$ in the system, the relation $u(1) \cdot v(1)=v(u(1))$ holds and in addition there are relations $r(1)=s(1)$ between certain pairs of additive words. Other properties of $L(S)$ are described in the following theorem.

TheOREM 7. The logarithmetic $L(S)$ of a subset $S$ of a groupoid $G$ has the following properties: (i) $L(S)$ is a groupoid with respect to addition and has a two-sided zero if $G$ has an identity, this zero also satisfying $0 \cdot x=x \cdot 0=0$ for all $x$, (ii) multiplication in $L(S)$ is associative, (iii) 1 , the generator of the additive groupoid $L^{+}(S)$ of $L(S)$, is a two-sided identity with respect to multiplication, (iv) $L(S)$ satisfies the distributive law $x(y+z)=x y+x z$, (v) $L^{+}(S)$ is the free monogenic $\mathfrak{B}$-groupoid for some subvariety $\mathfrak{B}$ of the variety of groupoids (or variety of groupoids with zero).

Proof. (i), (ii), (iii), (iv) have been discussed by Etherington, (v) follows from Theorem 2 after we have observed that $y \rightarrow x y$ is an endomorphism of $L^{+}(S)$ and thus there are endomorphisms of $L^{+}(S)$ which send the generator 1 into any element.

We define an abstract logarithmetic to be an algebraic system 
closed with respect to addition and multiplication and such that (i) it is a groupoid (possibly with a two-sided zero) with respect to addition, (ii) it possesses a two-sided identity with respect to multiplication and this element generates the additive groupoid, (iii) multiplication is associative, (iv) the distributive law $x(y+z)=x y+x z$ is satisfied. If the abstract logarithmetic has a zero, we assume that $x \cdot 0=0 \cdot x=0$ for all $x$.

Let $F_{1}(\mathfrak{B})$ be the free monogenic $\mathfrak{B}$-groupoid where $\mathfrak{B}$ is a subvariety of $\mathfrak{Z}$. We assume $F_{1}(\mathfrak{B})$ is written additively with generator 1. A multiplication $x \cdot y$ is introduced into $F_{1}(\mathfrak{B})$ by $x \cdot y=y \phi_{x}$ where $\phi_{x}$ is the endomorphism of $F_{1}(\mathfrak{B})$ which maps 1 onto $x$. The resulting system is an abstract logarithmetic which we shall denote by $L_{1}(\mathfrak{B})$. Corresponding to Theorem 4 we have the following theorem for abstract logarithmetics.

THEOREM 8. Let $L$ be an abstract logarithmetic such that its additive groupoid $L^{+}$belongs to the variety $\mathfrak{B}$. Then the homomorphism of $F_{1}(\mathfrak{B})$ onto $L^{+}$given by $1 \rightarrow 1$ can be extended to a homomorphism of $L_{1}(\mathfrak{B})$ onto $L$.

As a corollary of this we obtain the result due to Etherington, $[3$, p. 450$]$, that every abstract logarithmetic is a homomorphic image of $L_{1}(3)$. Combining the proof of Theorem 6 with part (v) of Theorem 7 we obtain the following stronger result.

THEOREM 9. Every abstract logarithmetic is isomorphic to an $L_{1}(\mathfrak{B})$, where $\mathfrak{B}$ is a subvariety or $\mathbb{B}$.

Using Theorem 3(a), we have the following theorem giving the number of abstract logarithmetics.

THEOREM 10. There are uncountably many nonisomorphic abstract logarithmetics.

The connection between the logarithmetics $L(S)$ and the abstract logarithmetics $L_{1}(\mathfrak{B})$ is given in the next theorem.

THEOREM 11. Let $r_{i}(x)=s_{i}(x), i=1,2,3, \cdots$, be the set of relations satisfied by every element in a subset $S$ of a multiplicative groupoid $G$. Let $\mathfrak{u}$ be the subvariety of $\mathbb{B}$ defined by the set of identical relations $r_{i}^{+}(x)=s_{i}^{+}(x), i=1,2,3, \cdots$, where $r_{i}^{+}(x), s_{i}^{+}(x)$ are $r_{i}(x), s_{i}(x)$ written additively. Then $L(S)$ is isomorphic to $L_{1}(\mathfrak{U})$.

Proof. The words $r_{i}(x), s_{i}(x)$ are powers of $x$ with exponents $r_{i}^{+}(1), s_{i}^{+}(1)$. Hence $L(S)$ can be considered as the homomorphic image of $L_{1}(\mathbb{B})$ obtained by adding the relations $r_{i}(1)=s_{i}(1)$. Now $L_{1}(\mathfrak{U})$ is 
the homomorphic image of $L_{1}(3)$ obtained by adding the identical relations $r_{i}(x)=s_{i}(x)$ to $L_{1}(3)$. These two homomorphic images of $L_{1}(3)$ are isomorphic since the relations $r_{i}(1)=s_{i}(1)$ in $L(S)$ imply that $L(S)$ satisfies the identical relations $r_{i}(x)=s_{i}(x)$.

CoRollaRy. If $G, H$ are two groupoids belonging to the variety $\mathfrak{B}$ and not to any subvariety of $\mathfrak{B}$, then $L(G), L(H), L_{1}(\mathfrak{B})$ are isomorphic.

In several papers (see [7] and the references given there) H. Popova has investigated the logarithmetics of finite loops and quasigroups. Because of this finiteness restriction only "positive" nonassociative integers are needed for exponents and the definition of these logarithmetics is exactly the same as the definition for groupoids. To discuss the logarithmetic of an arbitrary quasigroup or loop we need to introduce "negative" exponents. This can be done by introducing an additive notation for quasigroups and loops corresponding to the multiplicative definition in terms of three operations given in [4]. In that definition we replace $x \cdot y$ by $x+y, x \backslash y$ by $-x+y$, and $x / y$ by $x-y$. Then we use as exponents of elements in quasigroups or loops the elements of the free monogenic quasigroup or loop written additively with generator 1 . There is no difficulty in extending to this new algebra of exponents all the ideas introduced for logarithmetics of groupoids at the beginning of this section. Corresponding to Theorem 7 , we have the following.

THEOREM 12. If $Q$ is a loop, the logarithmetic $L(Q)$ of $Q$ is a left neoring with an identity which generates its additive loop.

We can also obtain theorems about logarithmetics of loops and quasigroups corresponding to Theorems 8, 9, 10 although in view of Theorem 12 and our earlier results about right neorings, these theorems are superfluous. As a consequence of the theorem corresponding to Theorem 11, the logarithmetics of two loops (quasigroups) are isomorphic if the loops (quasigroups) belong to the same subvariety. Another consequence of this theorem is that given any left neoring with a right identity such that this right identity generates the additive loop of the left neoring, there exists a loop having this left neoring as its logarithmetic. Thus the study of left or right neorings with an identity which generates the additive loop is equivalent to the study of logarithmetics of loops.

\section{REFERENCES}

1. Rafael Artzy, On loops with a special property, Proc. Amer. Math. Soc. vol. 6 (1955) pp. 448-453. 
2. R. H. Bruck, Analogues of the ring of rational integers, Proc. Amer. Math. Soc. vol. 6 (1955) pp. 50-57.

3. I. M. H. Etherington, Non-associative arithmetics, Proceedings of the Royal Society of Edinburgh vol. 62 (1949) pp. 442-453.

4. Trevor Evans, On multiplicative systems defined by generators and relations, I. Normal form theorems, Proc. Cambridge Philos. Soc. vol. 47 (1951) pp. 637-649.

5. - On multiplicative systems defined by generators and relations, II. Monogenic loops, Proc. Cambridge Philos. Soc. vol. 49 (1953) pp. 579-589.

6. Trevor Evans and B. H. Neumann, On varieties of groupoids and loops, J. London Math. Soc. vol. 28 (1953) pp. 342-350.

7. Helene Popova, Sur la logarithmetique d'une boucle, C.R. Acad. Sci. Paris vol. 236 (1953) pp. 1220-1222.

EMORY UNIVERSITY

\title{
COMPONENT SUBSETS OF THE FREE LATTICE ON $n$ GENERATORS
}

\author{
RICHARD A. DEAN
}

1. Introduction. In the free lattice on $n$ generators, $\mathrm{FL}(n)$, the components of a word (lattice polynomial) are defined recursively by: (i) the only component of a generator is itself, and (ii) if $W \equiv A \cup B$ (or $A \cap B$ ) the components of $W$ are $W, A$, and $B$ and their components. ${ }^{1}$ A component subset, $P$, of $\mathrm{FL}(n)$ is a collection of words in $\mathrm{FL}(n)$ with the following property: if a word belongs to $P$ then so do all its components. ${ }^{2}$ A component subset of $\mathrm{FL}(n)$ may be partially ordered in a natural way: $A \geqq B$ if and only if $A \geqq B$ in $\operatorname{FL}(n)$. Clearly if $W \equiv A \cup B$ (or $A \cap B$ ) belongs to a component subset, $P$, $W$ appears in $P$ as the l.u.b. (or g.l.b.) of $A$ and $B$ under the ordering $(\geqq)$. Thus it is natural to say that a component subset $P$ is generated by the generators of $\mathrm{FL}(n)$ which appear in the words belonging to $P$. This notion is used here to prove:

THEOREM $1 .^{8}$ Given any two words, unequal in $\mathrm{FL}(n)$, there exists a finite homomorphic image of $\mathrm{FL}(n)$ in which their images are distinct.

TheOREM 2.4 Any lattice possessing a countable number of generators

Received by the editors May 2, 1955 .

1 (三) denotes logical identity.

2 The author is indebted to R. P. Dilworth for suggesting the definition of component subset and its application in the proof of Theorem 2.

2 Theorem 1 was conjectured by Marshall Hall, Jr.

- Theorem 2 is due to Sorkin [4] where a sketch of a proof is given. 